\title{
Two-dimensional capillary origami
}

\author{
N. D. Brubaker ${ }^{\mathrm{a}}$, J. Lega $^{\mathrm{b}}$ \\ ${ }^{a}$ Department of Mathematics, University of Arizona, Tucson, Arizona, 85721, USA \\ nbrubaker@math.arizona.edu \\ ${ }^{b}$ Department of Mathematics, University of Arizona, Tucson, Arizona, 85721, USA \\ lega@math.arizona.edu
}

\begin{abstract}
We describe a global approach to the problem of capillary origami that captures all unfolded equilibrium configurations in the two-dimensional setting where the drop is not required to fully wet the flexible plate. We provide bifurcation diagrams showing the level of encapsulation of each equilibrium configuration as a function of the volume of liquid that it contains, as well as plots representing the energy of each equilibrium branch. These diagrams indicate at what volume level the liquid drop ceases to be attached to the endpoints of the plate, which depends on the value of the contact angle. As in the case of pinned contact points, three different parameter regimes are identified, one of which predicts instantaneous encapsulation for small initial volumes of liquid.
\end{abstract}

Keywords: elasto-capillary system, capillary origami, nonlinear plate, energy minimization

PACS: 68.08.-p 46.32.+x 81.16.Dn 85.85.+j

\section{Introduction}

Capillary origami, namely the folding of a flexible two-dimensional membrane into a predetermined three-dimensional shape through the action of surface capillary forces, is an example of micro-origami [1]. This technique (see [2] for a review), which also encompasses folding of planar structures by means of chemical [3], thermal [4], and electrostatic forces [5, 6], presents an interesting alternative to the traditional methods of micro-fabrication based on the deposition and etching of thin films $[7,8]$. Capillary origami is observed in nature [9] and its applications include lab-on-a-chip devices [10], graphene actuation [11] and self-assembly $[12,13,14]$.

Preprint submitted to Physics Letters A

August 31, 2015

(C) 2015. This manuscript version is made available under the Elsevier user license http://www.elsevier.com/open-access/userlicense/1.0/ 
The seminal article of Py et al. [15] provided an elegant proof of concept for capillary origami, showing that a variety of three-dimensional structures, such as cubes or pyramids, could be created by this method. A capillary origami system consists of a thin, flat two-dimensional plate, lying on a hydrophobic substrate, over which one places a small drop of liquid. Initially, the solid-liquid adhesive forces overcome the strength of elastic forces within the plate, deforming the system into an equilibrium position where the plate at least partially adheres to the drop of liquid. As the volume of liquid is reduced, for instance by evaporation $[16,17,18,19]$, the thin sheet can completely encapsulate the liquid, thereby forming a three-dimensional structure whose shape is predetermined by the initial cut out of the two-dimensional thin film. Alternatively, immediate folding may be achieved upon impact of a drop on a flexible membrane [20].

The purpose of this letter is to promote a unified and systematic approach to the problem of capillary origami and to apply this method to identify all two-dimensional equilibrium structures of a capillary origami system in the general situation where the liquid is not required to completely wet the flexible plate. The shape of the air-liquid interface, as well as the extent over which the liquid is in contact with the plate, are obtained as consequences of minimizing the total energy $E$ of the system, under appropriate constraints. This minimization is performed on the actual energy, as opposed to for instance a discretized version thereof [18]. As a consequence, we obtain the ordinary differential equations and boundary conditions that equilibria must satisfy. These are Equations (2), which we then solve numerically, using an Euler-Newton predictor-corrector method. The resulting equilibrium configurations depend on the physical properties of the fluid and of the plate, as well as on a single control parameter, which is the volume of the liquid.

We present our results in the form of bifurcation diagrams showing the level of encapsulation of various branches of equilibria as a function of the volume $V$ of liquid in the system, together with corresponding plots for $E$. These plots explain how the bifurcation diagrams of [21], obtained for configurations in which the liquid wets the entire plate, are amended when partial wetting is taken into account. We conclude that, as in [21] for fixed contact points, three parameter regimes need to be considered in the general case. 


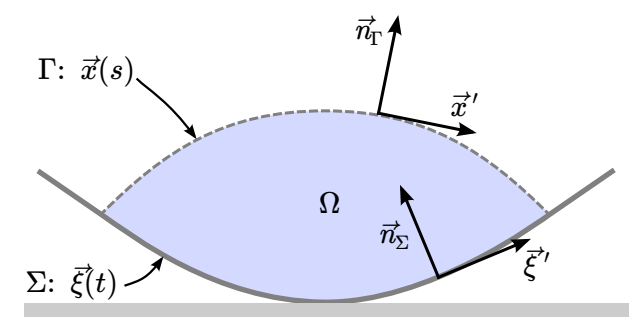

Figure 1: Two-dimensional capillary origami setup.

\section{Problem setup}

For simplicity, we neglect gravity and assume that the plate bends without stretching. These effects, as well as the presence of external forces generated for instance by an electric [22] or magnetic [23] field, may be included in the total energy of the system if needed. We suppose that all deformations are elastic, and we are only concerned with two-dimensional structures, that is three-dimensional configurations that are uniform by translation in one spatial direction. We therefore consider the two-dimensional capillary origami setup shown in Figure 1, consisting of a thin plate $\Sigma$ and a drop of liquid $\Omega$ whose interface with the surrounding air is given by the curve $\Gamma$. The volume $V$ of the drop of liquid (denoted as the area $A$ of the region delimited by $\Gamma$ and $\Sigma$ ), as well as the length $L$ of the plate, are fixed and finite. We look for configurations that are symmetric with respect to the vertical axis, but do not assume any particular shape for $\Gamma$ or $\Sigma$. These properties will be obtained as consequences of the minimization process.

To streamline the discussion, we explain our approach in the situation where the endpoints of $\Gamma$ and $\Sigma$ are in contact but give the final result in (2) for general configurations; an example of the latter is depicted in Figure 1. Details of the calculations are given in [21] for pinned contact points and summarized in the supplementary materials for non-pinned contact points. A longer article describing the calculations in the latter case is in progress and will be published elsewhere [24].

When the fluid wets the entire plate, the total energy of the system is comprised of the free surface energy of the liquid-air interface [25], and of the bending energy of the elastic plate [26]. This energy is given by $\gamma L E[\Gamma, \Sigma]$, where $\gamma \equiv \gamma_{L V}$ is the surface tension of the liquid-vapor interface $\Gamma$ and 
$E[\Gamma, \Sigma]$ is the dimensionless energy

$$
E[\Gamma, \Sigma]=\int_{\Gamma} d \sigma+\frac{1}{2 \lambda} \int_{\Sigma} \kappa_{\Sigma}^{2} d \sigma
$$

The elasto-capillary length of the system is given by $L_{e c}=L / \sqrt{\lambda}$ where $\lambda=\gamma L^{2} / B$ and $B$ is the bending rigidity of $\Sigma$. In (1), all lengths are relative to the length $L$ of the plate, $\kappa$ denotes the signed curvature of its subscript, and $d \sigma$ represents the arc-length element of the curve referred to in each integral. As detailed in [21], equilibrium configurations are obtained by calculating the first variation of the above energy assuming conservation of the volume of liquid $V$ and the length of the plate $L$. Using $\alpha$ and $\beta$ as Lagrange multipliers to account for these constraints, one must therefore minimize

$$
\mathcal{J}[\Gamma, \Sigma]=\int_{\Gamma} d \sigma+\frac{1}{2 \lambda} \int_{\Sigma} \kappa_{\Sigma}^{2} d \sigma+\alpha \int_{\Sigma} d \sigma+\beta \iint_{\Omega} d A
$$

To this end, we introduce perturbations $\Gamma_{\epsilon}$ and $\Sigma_{\epsilon}$ of the curves $\Gamma$ and $\Sigma$ parametrized as follows:

$$
\begin{array}{ll}
\Gamma_{\epsilon}: & \vec{x}_{\epsilon}(s)=\vec{x}(s)+\epsilon\left(u_{\Gamma}(s) \vec{n}_{\Gamma}(s)+v_{\Gamma}(s) \vec{x}^{\prime}(s)\right), \\
\Sigma_{\epsilon}: & \vec{\xi}_{\epsilon}(t)=\vec{\xi}(t)+\epsilon\left(u_{\Sigma}(t) \vec{n}_{\Sigma}(t)+v_{\Sigma}(t) \vec{\xi}^{\prime}(t)\right),
\end{array}
$$

where $\vec{x}(s)=(x(s), y(s))$ is a smooth parametrization of $\Gamma$ in terms of its arclength $s \in[-\ell, \ell]$ (here $\ell$ is the half-length of the air-liquid interface scaled to the length $L$ of the plate), $\vec{\xi}(t)=(\xi(t), \eta(t))$ is a similar parametrization of the plate by its arclength $t \in[-1 / 2,1 / 2]$, a prime represents the derivative with respect to arclength, $\vec{n}_{\Gamma}(s)=R_{\pi / 2} \vec{x}^{\prime}(s)$ and $\vec{n}_{\Sigma}(t)=R_{\pi / 2} \vec{\xi}^{\prime}(t)$ are the corresponding unit normals pointing out of and into the liquid respectively, and $R_{\omega}$ is the standard two-dimensional counter-clockwise rotation matrix of angle $\omega$ (see Figure 1). We do not assume that these perturbations are symmetric with respect to the vertical axis. The tangent and normal vectors for the perturbed air-liquid interface are related by the Serret-Frénet formulas

$$
\left(\frac{\vec{x}_{\epsilon}^{\prime}}{\left\|\vec{x}_{\epsilon}^{\prime}\right\|}\right)^{\prime}=\kappa_{\Gamma_{\epsilon}} \vec{n}_{\Gamma_{\epsilon}}\left\|\vec{x}_{\epsilon}^{\prime}\right\|, \quad \vec{n}_{\Gamma_{\epsilon}}^{\prime}=-\kappa_{\Gamma_{\epsilon}} \vec{x}_{\epsilon}^{\prime} ;
$$

similar relations also hold for the tangent and normal vectors for the perturbed plate. Requiring that the endpoints of $\Gamma_{\epsilon}$ are the same as the endpoints 
of $\Sigma_{\epsilon}$ leads to expressions for the tangential components of the perturbations,

$$
\begin{aligned}
v_{\Gamma}( \pm \ell) & =\frac{\vec{\xi}^{\prime} \cdot \vec{x}^{\prime}}{\vec{n}_{\Gamma} \cdot \vec{\xi}^{\prime}} u_{\Gamma}+\left.\frac{1}{\vec{n}_{\Sigma} \cdot \vec{x}^{\prime}} u_{\Sigma}\right|_{P_{ \pm}}, \\
v_{\Sigma}( \pm 1 / 2) & =\frac{1}{\vec{n}_{\Gamma} \cdot \vec{\xi}^{\prime}} u_{\Gamma}+\left.\frac{\vec{x}^{\prime} \cdot \vec{\xi}^{\prime}}{\vec{n}_{\Sigma} \cdot \vec{x}^{\prime}} u_{\Sigma}\right|_{P_{ \pm}},
\end{aligned}
$$

where $P_{ \pm}$denotes the points $(s, t)= \pm(\ell, 1 / 2)$. The equilibrium configurations, which are extrema of $\mathcal{J}[\Gamma, \Sigma]$, are obtained by calculating the derivative of $\mathcal{J}\left[\Gamma_{\epsilon}, \Sigma_{\epsilon}\right]$ with respect to $\epsilon$ at the value $\epsilon=0$ and demanding that this derivative vanish for all perturbations $u_{\Gamma}, u_{\Sigma}$. This leads to the following equations for the curvatures $\kappa_{\Gamma}$ and $\kappa_{\Sigma}$ of $\Gamma$ and $\Sigma$ :

$$
\begin{aligned}
& \kappa_{\Gamma}=\beta, \quad-\ell<s<\ell \\
& \kappa_{\Sigma}^{\prime \prime}+\frac{\kappa_{\Sigma}^{3}}{2}-\alpha \lambda \kappa_{\Sigma}=\beta \lambda, \quad-\zeta<t<\zeta,
\end{aligned}
$$

with boundary conditions

$$
\kappa_{\Sigma}=0, \quad \kappa_{\Sigma}^{\prime}=\lambda \vec{n}_{\Sigma} \cdot \vec{x}^{\prime}, \quad \vec{\xi}^{\prime} \cdot \vec{x}^{\prime}+\alpha=0
$$

at the contact points $(s, t)= \pm(\ell, \zeta)$. The parameter $\zeta$ is equal to $1 / 2$ for pinned contact points. As explained in the supplementary material and detailed in [24], the above equations extend to the case of free contact points, for which $0<\zeta<1 / 2$, with the additional constraints $\alpha \geq-\tau$ and $(\alpha+$ $\tau)(2 \zeta-1)=0$, where $\tau$ is the known relative adhesion coefficient $\tau=\left(\gamma_{S V}-\right.$ $\left.\gamma_{L S}\right) / \gamma$

System (2a) immediately implies that the air-liquid interface $\Gamma$ is, as expected, an arc of a circle and, thus, reduces to a single differential equation for $\kappa_{\Sigma}$. We showed in [21] that in the case of pinned contact points this is equivalent to the model proposed by Py et al. in [15], which is also used in [27] in the idealized case of a plate of infinite length. To the best of our knowledge, the above equations are new for non-pinned contact points. Their significance is detailed in the bifurcation diagrams of the next section.

\section{Bifurcation Diagrams}

In this section, we present bifurcation diagrams that describe all basic equilibrium configurations of a two-dimensional capillary origami system. 
These diagrams, which complete and extend the diagrams of [15] and [21] to the case of non-pinned contact points, are obtained by numerically solving system (2), allowing the parameter $\zeta$ to be less than $1 / 2$, with the appropriate constraints on $\alpha$ and $\beta$ mentioned above. These diagrams show the distance across the opening of the plate $\xi(1 / 2)-\xi(-1 / 2) \equiv 2 \xi(1 / 2)$ as a function of the volume $V$ of liquid in the system. An important result of [21] is that three situations, associated with different parameter ranges for $\lambda$, need to be considered. The numerical values of the thresholds $\lambda_{*}$ and $\lambda^{*}$ that define these ranges are given in [21] for pinned contact points. A conclusion of the present work is that these values depend on the contact angle $\theta$ in the non pinned case but the general structure does not.

For $\lambda<\lambda_{*}$, Figure 2 indicates that following the top branch from large to small values of $A$ (as illustrated in the movie lam12.mp4 provided as supplemental material) does not lead to encapsulation, since the plate starts to bend under the action of capillary forces but then opens up again as the amount of liquid (measured by $A$ ) gets close to zero. A second branch leading to full encapsulation exists but is of higher energy.

For intermediate values of $\lambda$, such that $\lambda_{*}<\lambda<\lambda^{*}$, the branch that exists for large values of $A$ continuously leads to encapsulation as $A$ is decreased (see the movie lam15.mp4). However, as shown in Figure 3, the latter configurations are metastable, as a second branch corresponding to an open structure exists for small values of $A$, and has lower energy. An almost-folded configuration could therefore snap open as the liquid evaporates.

For values of $\lambda$ larger than a second threshold $\lambda^{*}$, there is a range of values of $A$ where the folded configuration is the only existing solution (see Figure 4 and movie lam50.mp4). In this case, capillary forces are strong enough to completely fold the plate as the volume of the drop is reduced, and the resulting configuration is stable. Moreover, provided the right amount of liquid is initially put on the plate, the structure is expected to instantaneously fold into its only stable configuration, thereby providing a convenient way to quickly fold an elastic membrane by means of capillary effects. Equilibrium configurations for values of $A$ in the shaded region of the top panel of Figure 4 correspond to the folded and racket shapes shown in the inset, as discussed in [21] for the case of pinned boundary conditions. We expect similar structures to exist when the liquid is not assumed to wet the entire surface of the plate.

Configurations with large $A$ always correspond to a fully wetted plate and therefore are not affected by the value of the liquid contact angle. Dewetting occurs only at small $A$, and the configurations we observe there 

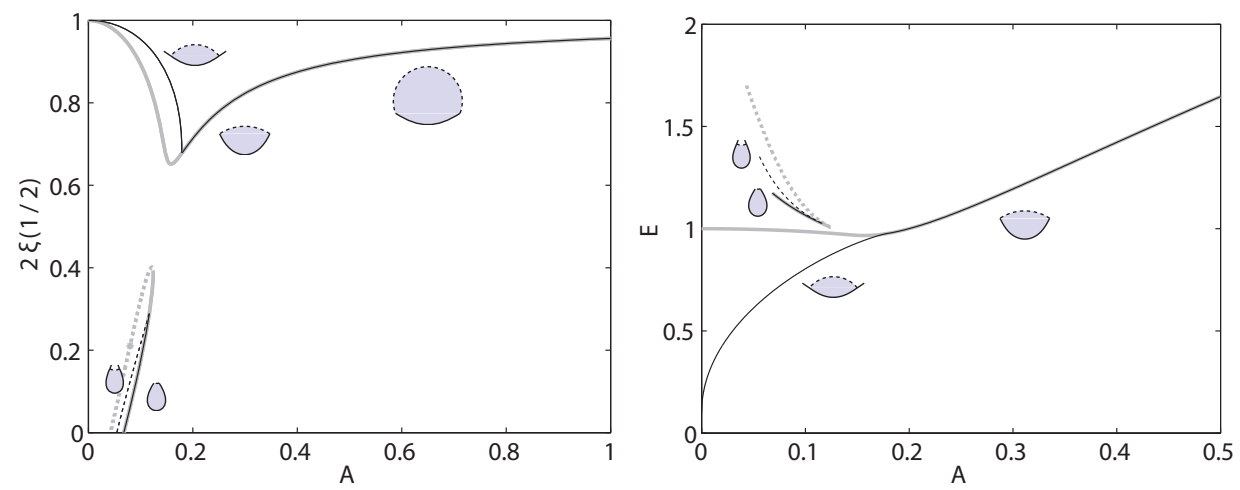

Figure 2: Left: bifurcation diagram for $\lambda=12$, which is typical of the first regime, defined by $\lambda<\lambda_{*}$. The control parameter $A$ represents the volume of liquid in the two-dimensional structure and $2 \xi(1 / 2)$ is a measure of the plate folding. Branches of equilibrium solutions with the liquid pinned at the endpoints of the plate are in grey (and in agreement with those obtained in [15]); those in which the drop is allowed to only cover part of the plate are in black. The value of $A$ where black branches detach from grey branches depends on the contact angle $\theta$, which is set at $\theta=\arccos (1 / 10) \simeq 84^{\circ}$. In both cases, solid curves denote configurations of lower energy along a given branch, and dashed curves those of higher energy. Right: energy $E$ of each of the equilibrium branches shown in the left panel. In both plots, icons representing the shape of the system are displayed along equilibrium branches.

are in agreement with those found in [27] in the case of plates of infinite length. Capillary origami experiments are typically performed with water drops on polydimethylsyloxane (PDMS) sheets $[15,20]$. Typical drop volumes are 1-100 microliters [15]. The order of magnitude of $\lambda=\gamma L^{2} / B$, where $B=E h^{3} /\left(12\left(1-\nu^{2}\right)\right), E$ is Young's modulus, $\nu$ the Poisson ratio, and $h$ the thickness of the plate, may be estimated as follows. For PDMS, $E \simeq 1$ $\mathrm{MPa}$ and $\nu \simeq 0.5$ [28], which gives for a plate length $L=2 \mathrm{~mm}$ and thickness $h=50 \mu \mathrm{m}[15], \lambda \simeq 21$. By vaying $L, h$, or $E$, values of $\lambda$ in the $10-200$ range should be attainable. In the fully wetted (FW) case, the critical values of $\lambda$ predicted by the theory are $\lambda_{*}^{F W} \simeq 12.54[21,15]$ and $\lambda_{F W}^{*} \simeq 24.05$ [21]. Experiments described in [15] estimate $\lambda_{*} \simeq 49$ for squares and $\lambda_{*} \simeq 142$ for triangles; these values are higher than $\lambda_{*}^{F W}$. The results presented here show that taking into account non-fully wetted solutions, which is more realistic, increases the value of $\lambda_{*}$ and therefore improves theoretical predictions. For instance, for a contact angle of $110^{\circ}$ which is typical for water on PDMS [20], we find that $\lambda_{*} \simeq 17.61$. A plot of $\lambda_{*}$ as a function of the contact angle $\theta$ is 

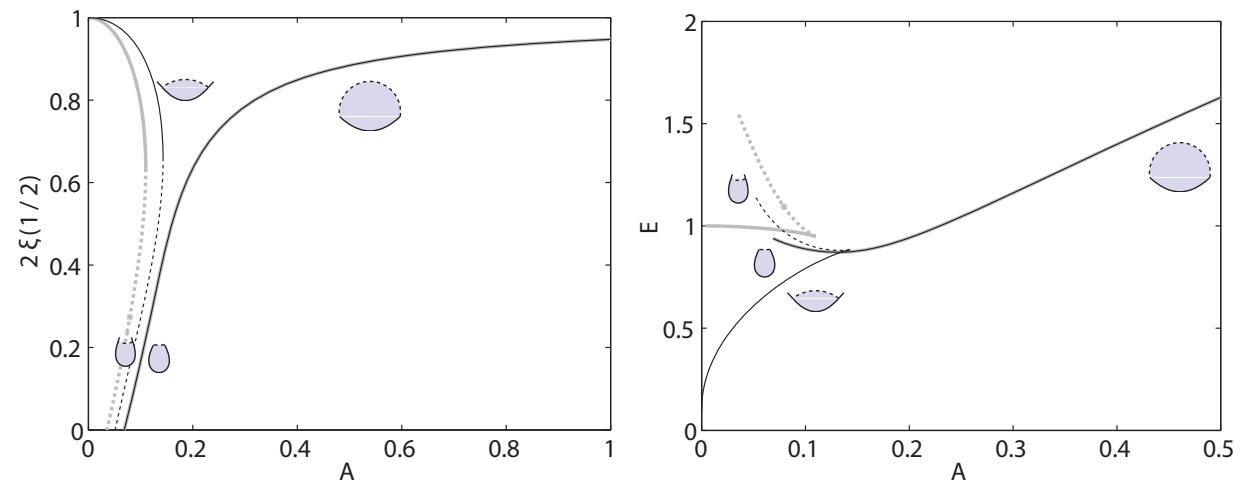

Figure 3: Same as Figure 2 but for $\lambda=15$, which is typical of the second regime, defined by $\lambda_{*}<\lambda<\lambda^{*}$.

given in Figure 5.

\section{Conclusions}

The conclusions of this work are twofold. First, the energetic approach described here provides a convenient framework to systematically determine all equilibrium branches of a two-dimensional capillary origami system consisting of a flexible membrane and a drop of liquid, and allows for the addition of external conservative forces when relevant. As in [15], no plastic deformations are considered. Examples of energy approaches for "crease origami" and buckling shells include [29] and [30] respectively.

The variational framework discussed here can in principle be extended to three dimensional systems, which should lead to further improvements on theoretical predictions for the critical values $\lambda_{*}$ and $\lambda^{*}$. In particular, one may take advantage of methods that estimate geometric quantities on triangulated manifolds [31] in order to approximate the total energy of the system and numerically find its minimizers. Moreover, it is shown in [24] that differential equations (2a) describing the curvature of the plate may also be obtained by demanding that deformations be isometric at lowest order. This fact therefore suggests a natural setup for three-dimensional problems in which the plate is only allowed to bend.

Second, our results indicate that for flexible enough membranes $\left(\lambda>\lambda^{*}\right)$, instantaneous encapsulation may occur, provided the correct amount of liquid is deposited on the plate. For more rigid membranes $\left(\lambda_{*}<\lambda<\lambda^{*}\right)$, even 

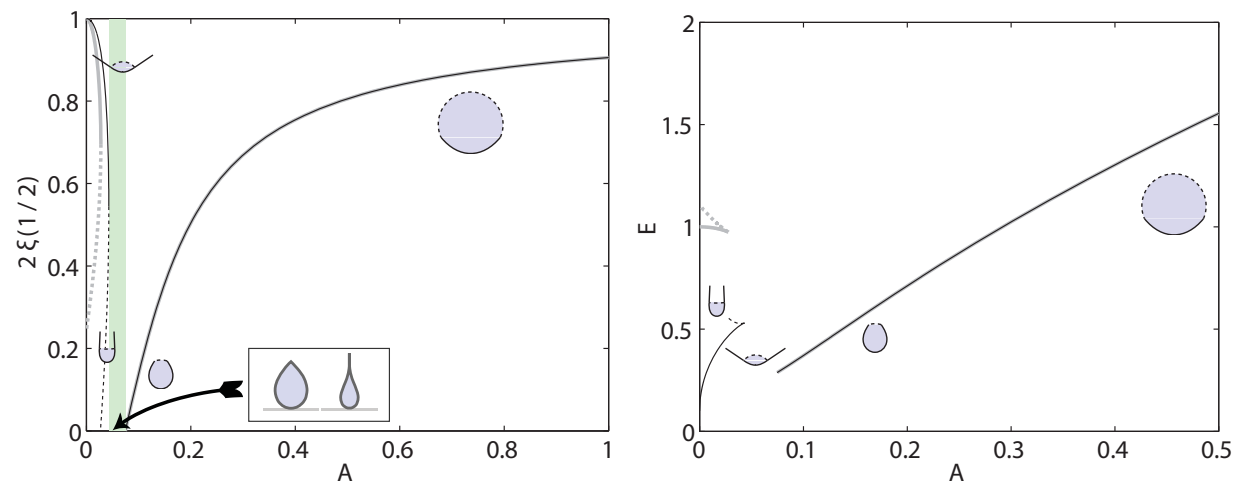

Figure 4: Same as Figure 2 but for $\lambda=50$, which is typical of the third regime, defined by $\lambda^{*}<\lambda$. The existence of this third regime was pointed out in [21] for pinned contact points. This figure shows that this regime, in which immediate encapsulation is expected as soon as the right amount of liquid is deposited on the plate, also exists when partial dewetting is included in the model. The inset shows fully wetted configurations for values of $A$ in the shaded region of the top panel [21].

if the encapsulated configuration can be reached by evaporation, it is not of minimum energy, and is therefore prone to reopening if destabilized.

\section{Acknowledgments}

This material is based upon work supported by the National Science Foundation under Award No. 1304090.

\section{References}

[1] S. T. Brittain, O. J. A. Schueller, H. Wu, S. Whitesides, G. M. Whitesides, Microorigami: Fabrication of small, three-dimensional, metallic structures, J. Phys. Chem. B 105 (2) (2000) 347-350.

[2] B. Roman, J. Bico, Elasto-capillarity: deforming an elastic structure with a liquid droplet, J. Phys. Condens. Matter 22 (49) (2010) 493101.

[3] J. M. Z. Ocampo, P. O. Vaccaro, T. Fleischmann, T.-S. Wang, K. Kubota, T. Aida, T. Ohnishi, A. Sugimura, R. Izumoto, M. Hosoda, S. Nashima, Optical actuation of micromirrors fabricated by the microorigami technique, Appl. Phys. Lett. 83 (18) (2003) 3647-3649. 


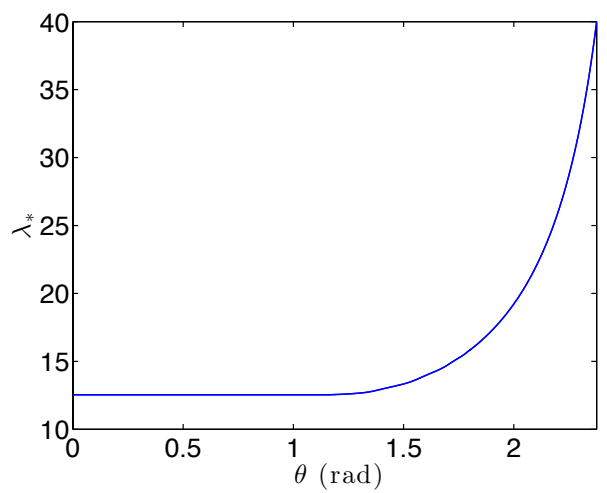

Figure 5: Plot of critical value $\lambda_{*}$ as a function the contact angle $\theta$. Note that $\lambda_{*}(\theta)$ is flat for $\theta \leq \theta_{*}$, where $\theta_{*} \simeq 1.15$ radians $\simeq 66^{\circ}$, and then increases for $\theta>\theta_{*}$.

[4] R. R. A. Syms, E. M. Yeatman, V. M. Bright, G. M. Whitesides, Surface tension-powered self-assembly of microstructures - The state-of-the-art, J. Microelectromech. Syst. 12 (4) (2003) 387-417.

[5] H. Okuzaki, T. Saido, H. Suzuki, Y. Hara, H. Yan, A biomorphic origami actuator fabricated by folding a conducting paper, J. Phys. Conf. Ser. 127 (1) (2008) 012001.

[6] E. Smela, O. Inganäs, I. Lundström, Controlled folding of micrometersize structures, Science 268 (5218) (1995) 1735-1738.

[7] G. T. A. Kovacs, K. Petersen, M. Albin, Peer reviewed: Silicon micromachining: Sensors to systems, Anal. Chem. 68 (13) (1996) 407A-412A.

[8] M. Madou, Fundamentals of Microfabrication: The Science of Miniaturization, 2nd Edition, CRC Press, Boca Raton, FL, 2002.

[9] S. Jung, P. M. Reis, J. James, C. Clanet, J. W. M. Bush, Capillary origami in nature, Phys. Fluids 21 (2009) 091110.

[10] A. J. de Mello, DNA amplification: Does "small" really mean "efficient"?, Lab Chip 1 (2) (2001) 24N-29N.

[11] N. Patra, B. Wang, P. Král, Nanodroplet activated and guided folding of graphene nanostructures, Nano Lett. 9 (11) (2009) 3766-3771. 
[12] M. Boncheva, D. A. Bruzewicz, G. M. Whitesides, Millimeter-scale selfassembly and its applications, Pure Appl. Chem. 75 (5) (2003) 621-630.

[13] J.-H. Cho, A. Azam, D. H. Gracias, Three dimensional nanofabrication using surface forces, Langmuir 26 (21) (2010) 16534-16539.

[14] J.-H. Cho, T. James, D. H. Gracias, Curving nanostructures using extrinsic stress, Adv. Mater. 22 (21) (2010) 2320-2324.

[15] C. Py, P. Reverdy, L. Doppler, J. Bico, B. Roman, C. N. Baroud, Capillary origami: spontaneous wrapping of a droplet with an elastic sheet, Phys. Rev. Lett. 98 (15) (2007) 156103-156103.

[16] E. de Langre, C. N. Baroud, P. Reverdy, Energy criteria for elastocapillary wrapping, J. Fluids Struct. 26 (2) (2010) 205-217.

[17] C. Py, P. Reverdy, L. Doppler, J. Bico, B. Roman, C. N. Baroud, Capillarity induced folding of elastic sheets, Eur. Phys. J. Special Topics 166 (2009) 67-71.

[18] M. Rivetti, S. Neukirch, Instabilities in a drop-strip system: A simplified model, Proc. R. Soc. London Ser. A 468 (2012) 1304-1324.

[19] J. W. van Honschoten, J. W. Berenschot, T. Ondarçuhu, R. G. P. Sanders, J. Sundaram, M. Elwenspoek, N. R. Tas, Elastocapillary fabrication of three-dimensional microstructures, Appl. Phys. Lett. 97 (1) (2010) 014103.

[20] A. Antkowiak, B. Audoly, C. Josserand, S. Neukirch, M. Rivetti, Instant fabrication and selection of folded structures using drop impact, Proc. Natl. Acad. Sci. USA 108 (26) (2011) 10400-10404.

[21] N. D. Brubaker, J. Lega, Two-dimensional capillary origami with pinned contact line, SIAM J. Appl. Math. 75 (3) (2015) 1275-1300.

[22] M. Piñeirua, J. Bico, B. Roman, Capillary origami controlled by an electric field, Soft Matter 6 (2010) 4491-4496.

[23] T. Jamin, C. Py, E. Falcon, Instability of the origami of a ferrofluid drop in a magnetic field, Phys. Rev. Lett. 107 (2011) 204503. 
[24] N. D. Brubaker, Two-dimensional capillary origami with inextensibility and a free contact line, Preprint.

[25] R. Finn, Equilibrium capillary surfaces, Vol. 284 of Grundl. Math. Wiss., Springer-Verlag, New York, 1986.

[26] L. D. Landau, E. M. Lifshitz, Theory of elasticity, Translated from the Russian by J. B. Sykes and W. H. Reid, 3rd Edition, Vol. 7 of Course Theoret. Phys., Pergamon Press, Oxford, UK, 1986.

[27] J. P. Péraud, E. Lauga, Geometry and wetting of capillary folding, Phys. Rev. E 89 (2014) 043011.

[28] I. D. Johnston, D. K. McCluskey, C. K. L. Tan, M. C. Tracey, Mechanical characterization of bulk sylgard 184 for microfluidics and microengineering, J. Micromech. Microeng. 24 (2014) 035017.

[29] M. A. Dias, L. H. Dudte, L. Mahadevan, C. D. Santangelo, Geometric mechanics of curved crease origami, Phys. Rev. Lett. 109 (2012) 114301.

[30] S. Knoche, J. Kierfeld, Buckling of spherical capsules, Phys. Rev. E 84 (2011) 046608.

[31] M. Meyer, M. Desbrun, P. Schroderl, A. H. Barr, Discrete differentialgeometry operators for triangulated 2-manifolds, in: H. C. Hege, K. Polthier (Eds.), Visualization and Mathematics III, Springer-Verlag Berlin Heidelberg, 2003, pp. 35-57. 